\title{
Attempting to reduce regurgitation and reingestion in a captive chimpanzee through increased feeding opportunities: a case study
}

\author{
Katie Struck, BS, Elaine N. Videan, PhD, Jo Fritz \& James Murphy
}

Persistent regurgitation and reingestion is an abnormal behavior seen in many captive NHPs, particularly gorillas and chimpanzees. Successful reduction of regurgitation in captive gorillas has been achieved by providing browse, such as branches and leaves, and feeding continuously throughout the day. However, this modality has not been tested in chimpanzees. The authors tested these methods in a 15-year-old male chimpanzee (Pan troglodytes) with a seven-year history of regurgitation and reingestion by alternately providing additional opportunities to forage or giving daily browse. The results show that providing browse on a daily basis models the feeding behavior of wild chimpanzees and is a successful way to reduce regurgitation and reingestion in a chimpanzee unresponsive to previous dietary changes.

Regurgitation and reingestion is a common, but poorly understood, abnormal behavior seen in captive nonhuman primates (NHPs), specifically gorillas and chimpanzees $^{1,2}$. This behavior consists of voluntarily bringing up partially digested food and then reingesting $\mathrm{it}^{3}$. Research on regurgitation in captive apes suggests the behavior may result from psychological issues (boredom or stress), housing conditions (lack of foraging materials or social stimulation), or feeding routines (lack of continual feeding, limited feeding periods, low fiber diet) $)^{1,2,4,5}$. In humans, frequent regurgitation has been shown to cause serious health problems, including esophageal motor disorders, periodontal disease, reflux esophagitis, and intestinal obstruction ${ }^{6}$. Therefore, reducing regurgitation among captive apes is recommended for their health and well-being.

Successful reduction of regurgitation in captive gorillas has been achieved by removing fruit from their diet and replacing it with high fiber vegetables that increase satiation and, presumably, suppress the motivation to regurgitate ${ }^{2}$. In addition, providing browse (foliated branches) and feeding continuously throughout the day have both been shown to successfully reduce regurgitation in captive gorillas ${ }^{2,7}$. One study of captive chimpanzees also suggested that continuously providing edible material (straw, forage, browse) may reduce regurgitation ${ }^{1}$. Wild chimpanzees spend between 30\% and $60 \%$ of their daily activity budget feeding and foraging $^{8-10}$, which contrasts sharply with the practice of providing captive chimpanzees with $2-3$ discrete meals per day ${ }^{1}$. That chimpanzees feed continuously in their native habitat is itself indirect evidence that adopting a continuous-feeding regime for captive chimpanzees may alleviate or eliminate regurgitation, but no study has directly tested this hypothesis in captive chimpanzees.

This case study focuses on a 15-year-old adult male chimpanzee housed at the Primate Foundation of Arizona (PFA) and whose regurgitation and reingestion had been particularly unresponsive to dietary adjustments (replacement of fruit with high fiber vegetables). This male was previously treated for nine months with a selective serotonin re-uptake inhibitor, during which there was some reduction in regurgitation but no consistent amelioration of the behavior. Our study specifically tested the continuous-feeding hypothesis for the reduction of regurgitation; we predicted that providing 
increased foraging opportunities and access to browse material would reduce the chimpanzee's regurgitation.

\section{METHODS}

Data was collected at the PFA, a nonprofit research and housing facility accredited by the Association for Assessment and Accreditation of Laboratory Animal Care (AAALAC) International. Methods were approved by the PFA Institutional Animal Care and Use Committee.

Housing consisted of indoor and outdoor areas that exceed current housing guidelines ${ }^{11}$. Social groups were housed indoors with outside access every other day. Each social group had an indoor enclosure that consisted of three interconnected cages providing 56.5 square meters of floor space and 2.88 meters of vertical height. Outdoor enclosures provided a floor space of 76.7 square meters and vertical space of 6.3 meters.

The subject for this case study was a 15-year-old mother-reared male chimpanzee with a seven-year history of regurgitation. Biannual physical examinations, dental evaluations, and hematology and serum chemistry analyses suggested the subject had been in good physical condition, despite the regurgitation behavior. The subject was housed with four other adult males in a stable social group and was middle-ranking in terms of dominance. Diet, adjusted to eliminate fruit, consisted of a morning vegetable meal, a midday monkeybiscuit meal, a mid-afternoon vitamin 'cocktail' (1 g sodium ascorbate dissolved in $6 \mathrm{oz}$ of Kool-Aid), an evening vegetable meal, and varied food enrichment (air-popped popcorn, sunflower seeds, chicken scratch given sporadically throughout the day).

Instantaneous focal animal sampling was used to record activity at 15 -second intervals over 30-minute trials. Regurgitation bouts were recorded using alloccurrence sampling. The subject was observed for one

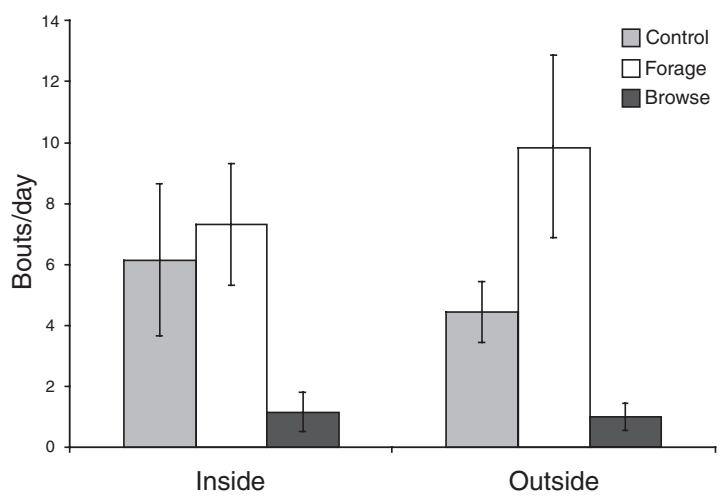

FIGURE 1 | Mean ( \pm standard error) number of regurgitation bouts per day during baseline, increased forage treatments, and increased browse treatments while subject was inside and outside. hour in the morning and one hour in the afternoon from March through April 2005. Observations were conducted Monday through Friday, both inside and outside, for six weeks.

During control (baseline) observations (11 h), the subject's diet and enrichment schedule remained unchanged. The two treatments, representing increased feeding opportunities, consisted of either forage or browse. During the forage observations (13.5 h), an extra meal of forage (unsweetened cereal, sunflower seeds, peanuts, popcorn, and chicken scratch) was distributed to the group approximately 10 minutes before the observation. During the browse observations (13 h), the group was supplied with browse (palm (Washingtonia robusta) fronds and stems, bamboo (Phyllostachys spp), Palo Verde (Cercidium microphyllum), and Ash (Fraxinus anomala) branches) approximately 10 minutes before the observation. Sufficient amounts of browse were distributed to the group, such that every individual had access. Both forage and browse treatments were randomized throughout the study, alternating between mornings and afternoons to control for time-of-day effects.

Data was summarized as the number of regurgitation bouts per observation (all-occurrence method) and the percentage of time (scans) the subject spent in regurgitation behavior (instantaneous sampling method). Data was analyzed using a Step-wise General Linear Model with the $P$-value set at 0.01 because of the small sample size. Time of day (morning or evening), location (inside or outside), and treatment phase (control, forage, or browse) were included as independent variables. Statistical analyses were conducted using Systat 10.2 and all tests were two-tailed.

\section{RESULTS}

The treatments did increase the percent of time (scans) spent foraging and feeding from $4.9 \%$ during baseline observations to $28.4 \%$ during forage treatments and $48.3 \%$ during browse treatments. Time of day (bouts: $\mathrm{F}=0.075, P=0.785$; time: $\mathrm{F}=0.168, P=0.683)$ and location (bouts: $\mathrm{F}=0.028, P=0.869$; time: $\mathrm{F}=0.342$, $P=0.561$ ) had no significant effect on either the number of bouts or percent time (scans) spent regurgitating and reingesting. However, treatment did have a significant effect $(\mathrm{F}=5.019, P=0.009)$ on the number of bouts per observation and a borderline significant effect ( $\mathrm{F}=3.581, P=0.033$ ) on percent time (scans) spent regurgitating and reingesting (Table 1 ).

On days when the subject was provided with browse, the average number of regurgitation bouts per hour decreased from 2.7 to 0.5 (Fig. 1). The percentage of time spent engaged in regurgitation behavior also decreased, dropping from $2.3 \%$ to $0.3 \%$ (Fig. 2). On days when the subject was provided with forage, the average number of regurgitation bouts per hour 
increased from 2.7 to 4.4 (Fig. 1). Percentage of time spent engaged in regurgitation behavior also increased, climbing from $2.3 \%$ to $4.2 \%$ (Fig. 2 ).

\section{DISCUSSION}

This case study demonstrates the promising results achieved when browse was made available to a habitually regurgitating chimpanzee, as suggested in previous studies of captive gorillas ${ }^{2,7}$. Although regurgitation was not totally eliminated, the decrease in the behavior was obvious. Expert estimates of the wild chimpanzee diet suggest that up to $35 \%$ of its total dry weight consists of fiber ${ }^{12-14}$. For this reason, captive NHP diets have, in the past, been criticized for not providing enough fiber ${ }^{15,16}$. The wild chimpanzee diet varies in composition, with individuals consuming approximately $25 \%$ of their diet as leaves, pith, bark, and terrestrial herbaceous vegetation $^{8,12,17,18}$. Bamboo is estimated to be composed of $75 \%$ fiber ${ }^{19}$, while Palo Verde contains 35\% fiber ${ }^{20}$. Both plants represent much higher fiber content than the typical captive chimpanzee $\operatorname{diet}^{21}$. The captive diet is similar to the wild chimpanzee diet in terms of daily diversity; however, the bulk of the dry weight and caloric intake comes from the monkey-biscuit meal, generally much less fibrous $(\sim 5-10 \%)$ than the equivalent for wild chimpanzees. By including browse in the captive chimpanzee diet, we can more closely approximate the wild chimpanzee diet and increase dietary fiber content. It is probable that the greater fiber content provided by the browse increased satiation, resulting in reduced regurgitation.

Increasing the foraging opportunities available to the subject had the opposite effect of that predicted. When the subject received forage, the mean number of bouts increased. Whether the forage was provided with or without any material to cover it, such as straw and sawdust, the subject ingested the forage within approximately 20 minutes (more quickly than the browse). It is possible that this led to less satiation and therefore regurgitation. These results are in surprising contrast to those of other chimpanzees ${ }^{22}$. Baker ${ }^{22}$ found that the introduction of straw bedding, cardboard, fruit peels, and scattered forage reduced regurgitation behavior

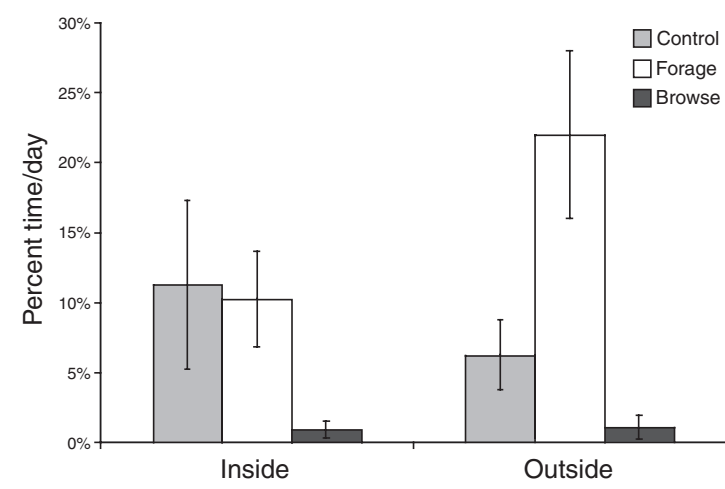

FIGURE 2 | Mean ( \pm standard error) percent time (scans) spent in regurgitation per day during baseline, increased forage treatments, and increased browse treatments while subject was inside and outside.

in individual chimpanzees by two-thirds. However, the introduction of forage and bedding was novel in those subjects, whereas the PFA chimpanzees have consistently received bedding and forage. Baker ${ }^{22}$ added cardboard and fruit peels as 'wadge' material, originally defined by Goodall ${ }^{9}$ as plant or fibrous material that is crushed with molars or peeled with incisors, chewed, sucked, and then discarded. It is likely that the novelty of the bedding and wadge material (cardboard, fruit peels) influenced the reduction in regurgitation, and that the effect did not result from the addition of forage material alone.

Continuous all-day feeding as a deterrent to regurgitation, as suggested in some articles, is difficult to achieve in practice. This study shows that increasing the number of feeding periods through additional daily forage was not successful in curbing regurgitation and reingestion, but actually encouraged the behavior. Results indicated that a more appropriate way to substitute for a continuous feeding pattern is to provide long-lasting edible material in the form of browse. Wild chimpanzees spend considerable amounts of time chewing, manipulating, and wadging the fibrous fruits, leaves, pith, and herbaceous vegetation in their diets 9 .

TABLE 1 | Effects of time of day, location, and treatment on mean (+/- standard deviation) number of regurgitation bouts and percent time (scans) spent regurgitating.

\begin{tabular}{llll} 
Variable & Subcategory & Number bouts & Percent time \\
\hline Time of day & Morning & $2.08 \pm 2.02$ & $3.95 \pm 0.04$ \\
& Afternoon & $0.46 \pm 2.63$ & $0.56 \pm 0.07$ \\
\hline Location & Inside & $2.05 \pm 2.02$ & $3.91 \pm 0.04$ \\
& Outside & $0.44 \pm 2.63$ & $0.51 \pm 0.07$ \\
\hline Treatment & Control & $1.36 \pm 2.24^{*}$ & $2.31 \pm 0.05^{*}$ \\
& Browse & $0.27 \pm 0.60^{*}$ & $0.26 \pm 0.01^{*}$ \\
& Forage & $2.19 \pm 3.01^{*}$ & $4.20 \pm 0.08^{*}$
\end{tabular}


In this study, branches, leaves, stalks, and stems were manipulated and chewed much longer than the forage, more closely approximating the feeding and wadging behavior of wild chimpanzees.

Observations from this study and from the subject's caregivers suggest that regurgitation is most common following meals. The most successful way to reduce or possibly eliminate regurgitation in this individual would be to give browse after every meal. This would prolong the subject's feeding period and provide extra fiber that may make regurgitation of the meal more difficult. Future research on the timing of the provision of browse (after feeding, for instance) may lead to more information about treating regurgitation behavior.

\section{ACKNOWLEDGMENTS}

The authors thank all PFA care staff for their help. This study was funded in part by University of Texas, M.D. Anderson Cancer Research Center, as a subcontract (1U42RR15090-05) within the National Institutes of Health (NIH) Biomedical Research Program.

\section{COMPETING INTERESTS STATEMENT}

The authors declare that they have no competing financial interests.
Received 3 August 2006; accepted 27 September 2006. Published online at http://www.labanimal.com.

1. Baker, K. \& Easley, S. An analysis of regurgitation and reingestion in captive chimpanzees. Appl. Anim. Behav. Sci. 49(4), 403-415 (1996).

2. Lukas, K.E. A review of nutritional and motivational factors contributing to the performance of regurgitation and reingestion in captive lowland gorillas (Gorilla gorilla gorilla). Appl. Anim. Behav. Sci. 63(3), 237-249 (1999).

3. Morgan, L., Howell, S.M. \& Fritz, J. Regurgitation and reingestion in a captive chimpanzee (Pan troglodytes). Lab Anim. (NY) 22(8), 42-45 (1993).

4. Akers, J.S. \& Schildkraut, D.S. Regurgiation/reingestion and coprophagy in captive gorillas. Zoo Biol. 4(2), 99-109 (1985).

5. Howell, S.M., Fritz, J., Downing, S. \& Bunuel, M. Treating chronic regurgitation behavior: a case study. Lab Anim. (NY) 26(2), 30-33 (1997).

6. O'Brien, M.D., Bruce, B.K. \& Camilleri, M. The rumination syndrome: clinical features rather than manometric diagnosis. Gastroenterology 108(4), 1024-1029 (1995).

7. Ruempler, U. The Cologne Zoo diet for lowland gorillas to eliminate regurgitation and reingestion. Int. Zoo Yearb. 31, 225-229 (1992).

8. Wrangham, R.W. in Primate Ecology: Studies of Feeding and Ranging Behavior in Lemurs, Monkeys, and Apes (ed. CluttonBrock. T.H.) 503-538 (Academic Press, New York, 1977).

9. Goodall, J. The Chimpanzees of Gombe: Patterns of Behavior 237-240 (The Belknap Press of Harvard University Press, Cambridge, MA, 1986).

10. Boesch, C. \& Boesch, H. The Chimpanzees of Tai Forest (0xford: Oxford University Press, Oxford, UK, 2000).

11. Institute of Laboratory Animal Research, National Research Council. Guide for the Care and Use of Laboratory Animals (National Academy Press, Washington, DC, 1996).

12. Hladik, C.M. in Primate Ecology: Studies of Feeding and Ranging Behavior in Lemurs Monkeys and Apes (ed. Clutton-Brock, T.H.) 481-501 (Academic Press, New York, 1977).

13. Conklin-Brittain, N.L., Wrangham, R.W. \& Hunt, K.D. Dietary response of chimpanzees and cercopithecines to seasonal variation in fruit abundance: II. Macronutrients. Int. J. Primatol. 19(6), 971-997 (1998).

14. Takemoto, H. Phytochemical determination for leaf food choice by wild chimpanzees in Guinea, Bossou. J. Chem. Ecol. 29(11), 2551-2573 (2003).

15. Crissey, S.D. \& Pribyl, L.S. Utilizing wild foraging ecology information to provide captive primates with an appropriate diet. Proc. Nutr. Soc. 56(3), 1083-1094 (1997).

16. McGrew, W.C. \& Pruetz, J.D.E. in The Care and Management of Captive Chimpanzees (ed. Brent, L.) 17-38 (American Society of Primatologists, San Antonio, 2001).

17. Yamakoshi, G. Dietary responses to fruit scarcity of wild chimpanzees at Bossou, Guinea: possible implications for ecological important of tool use. Am. J. Phys. Anthropol. 106(3), 283-295 (1998).

18. Newton-Fisher, N.E. The diet of chimpanzees in the Budongo Forest Reserve, Uganda. Afr. J. Ecol. 37(3), 377-354 (1999).

19. National Research Council. Nutrient Requirements of Nonhuman Primates 2 nd edn. (National Academies Press, Washington, DC, 2003).

20. Ramirez, R.G. \& Lara, J.A. Influence of native shrubs Acacia rigidula, Cercidium macrum and Acacai farnesiana on digestibility and nitrogen utilization by sheep. Small Rumin. Res. 28(1), 39-45 (1998).

21. Callahan, R., Scarry, C., Howell, S., Schwandt, M. \& Fritz, J. Macro- and micro-nutrient content of the diet of sociallyhoused chimpanzees (Pan troglodytes) at the Primate Foundation of Arizona. Am. J. Primatol. 60(Suppl 1), 78 (2003).

22. Baker, K.C. Straw and forage material ameliorate abnormal behaviors in adult chimpanzees. Zoo Biol. 16(3), 225-236 (1997). 